\title{
Probiotics in Periodontology
}

\author{
Christina PrithikaSimon, Dr.Karthikeyan. \\ (Intern, Saveetha Dental college, Saveetha University, India) \\ (Senior Lecturer, Saveetha Dental college, Saveetha University,India)
}

\begin{abstract}
Probiotics has now become the field of interest of researchers. Time has come to shift the paradigm of treatment from specific bacteria elimination to altering bacterial ecology by probiotics. With increase in the incidence of resistance to antibiotics, probiotics maybe a promising area of research in periodontal therapy. Probiotics utilize naturally occurring bacteria to confer a healthy benefit when administered in adequate amounts.Probiotics provide us an alternative way to combat diseases and has been introduced for prevention and treatment of periodontal diseases.This article reviews the role of probiotics for management of periodontal diseases and explores its potential as a novel treatment strategy.
\end{abstract}

\section{Introduction}

The term probiotic is derived from the Greek word, meaning "for life." Probiotics are living organisms, principally bacteria that are safe for human consumption and when ingested in sufficient quantities, have beneficial effects on human health, beyond the basic nutrition ${ }^{\text {ee }}$. The interest in probiotics and the modulation of the microbiota for restoring and maintaining health have gained a lot of attention over the past decade. The term 'probiotic' is a relatively new word and is currently used to name bacteria with beneficial effects for humans and animals.

\section{Criteria For Probiotics}

To be considered for use as probiotic following criteria needs to be fulfilled.

1) It should capable of exerting a beneficial effect on the host animal.

2) It should be of human origin.

3) It should have high cell viability.

4) It should be non-pathogenic and non-toxic.

5) It should be able to interact or to send signals to immune cells.

6) It should have capacity to influence local metabolic activity.

7) It should be capable of surviving and metabolising in the gut environment.

8) It should be stable and capable of remaining viable for periods under storage and field conditions.

\section{Probiotics And Periodontal Disease}

Periodontal diseases are classified into two major types- gingivitis and periodontitis. Gingivitis is characterized by inflammation of gingiva, whereas periodontitis is a progressive, destructive disease that affects all supporting tissues of teeth, including the alveolar bone. The main pathogenic agents associated with periodontitis are Porphyromonasgingivalis, Treponemadenticola, Tannerellaforsythiaetc

\section{Inhibition of specific organisms}

\section{Mechanism Of Action}

Inhibition of pathogen adhesion, colonization and biofilm formation Inhibition of pathogen growth by various substances such as organic acids, hydrogen peroxide and bacteriocins against oral pathogens.

\section{Effects on host response}

Inhibition of collagenases and reduction of inflammation associated molecules Induction of expression of cytoprotective proteins on host cell surfaces Modulation of pro-inflammatory pathways induced by pathogens Prevention of cytokine-induced apoptosis Modulation of host immune response

\section{Clinical Parameters And Probiotics}

In the study by Twetman et al. (2009), 17 the authors observed that as soon as the probiotic intake was stopped, the percentage of sites that were bleeding upon probing positive, increased again. In the study of 
Twetman et al. (2009),17 the focus was predominantly on gingival inflammation and the production of pro and anti-inflammatory cytokines [IL-1b, tumour necrosis factor-a (TNF-a), IL-6, IL-8 and IL-10]. During the two weeks of intervention, the gingival crevicular fluid volume decreased significantly in the probiotic groups, whereas no significant changes were observed in the placebo group. The levels of TNF and IL-8 also decreased significantly after 1 and two weeks respectively in the probiotic group, which used the highest dose of probiotics, compared with baseline. However, these effects were only temporary and tended to return to baseline values two weeks after discontinuing the probiotics

\section{Effectiveness of Probiotics in Halitosis}

Halitosis or oral malodour refers to the foul and unpleasant odour emanating from the oral cavity. Volatile sulphur compounds (VSC) are responsible for halitosis. Bacteria responsible for VSC production are Fusobacteriumnucleatum, Porphyromonasgingivalis, Prevotellaintermedia, and Treponemadenticola. Streptococcus salivarius produces bacteriocins which inhibit bacteria producing VSC. It was shown that lozenges and gum containing Streptococcus salivarius decrease VSC in halitosis patients. The use of gum or lozenges containing S. salivarius reduced levels of volatile sulphur compounds among patients diagnosed with halitosis.

\section{Effectiveness of Probiotics in Yeast infections}

Elahi et al assessed the pattern of colonization of Lactobacillus acidophilus and Lactobacillus fermentum and demonstrated a rapid decline in Candida albicans after the intake of probiotics strains in mice. Continuous consumption of probiotics led to almost undetectable numbers of fungi in the oral cavity, maintaining the protective effect for a prolonged period after cessation of application.

\section{Safety Aspects Of Probiotics}

Increased probiotic supplementation of different food products during the recent years has raised safety concerns. When probiotics are applied orally, at least a part of them will be ingested and can interact with a patient's systemic health. When ingested orally, probiotics are generally considered safe and well tolerated with bloating and flatulence occurring frequently.

The increased probiotic consumption inevitably leads to increased concentrations of these species in the host organism. Although rare, cases of probiotics-related bacteraemia, lactobacillus endocarditis and liver abscess secondary to L. rhamnosus have been reported in the literature and such cases have responded well to appropriate antibiotic therapy.

\section{Criteria Of A Microorganismused As Probiotics}

High cell viability, resistant to low ph and acids

Ability to persist

Adhesion to cancel the flushing agent

Able to interact or to send signals to immune cells

Should be of human origin

Should be non pathogenic

Resistance to processing

Influence local metabolic activity

\section{Conclusion}

In the recent times when organisms are fast developing resistance to antibiotics, the emergence of probiotics appears to be a boon for the treatment of diseases. With fast evolving technology and integration of biophysics with molecular biology, designer probiotics poses huge opportunity to treat diseases in a natural and non-invasive way. Periodontitis has established risk of various systemic diseases like diabetes, atherosclerosis, pre-term low birth. Thus, a critical need to establish good periodontal health for attaining good systemic health is of utmost importance and probiotics are promising an increasingly positive effect. However, the effects of probiotics on periodontal health and its maintenance including means of administration, dosage and safety aspects are not clear. Numerous randomized clinical studies will be required to clearly establish the potential of probiotics in the prevention and treatment of periodontal diseases. There is no doubt that with further significant progress, probiotics may have an important role to play in the near future within the periodontal arena. 


\section{References}

[1]. Hamilton-Miller JMT, Gibson GR, Bruck W. "Some insights into the derivation and early uses of the word probiotic". Br J Nutr 2003 (90):845.

[2]. Report of a Joint FAO/WHO Expert consultation on evaluation of health and nutritional properties of probiotics in food including powder milk with live lactic acid bacteria (October 2001).

[3]. Brown AC, Valiere A. Probiotics and medical nutrition therapy. NutrClin Care 2004; 7:56-68.

[4]. Tanboga I, Caglar E, Kargul B. Campaign of probiotic food consumption in Turkish children, oral perspectives ,Probiotics for your child ${ }^{\text {ee }}$. Int J Pediatr Dent 2003; 13:59-64.

[5]. Teughels W, Loozen G, Quirynen M. Do probiotics offer opportunities to manipulate the periodontal oral microbiota? J ClinPeriodontol 2011;38(Suppl 11):159-77. Shimauchi H, Mayanagi G, Nakaya S, Minamibuchi

[6]. M, Ito Y, Yamaki K, et al. Improvement of periodontal condition by probiotics with lactobacillus salivarius WB21: A randomized, double-blind, placebo-controlled study. J ClinPeriodontol 2008;35:897-905.

[7]. Bo“hm SK, Kruis W. Probiotics: do they help to control intestinal inflammation? Annals of the New York Academy of Sciences 2006;1072:339-50

[8]. Teughels W, Newman MG, Coucke W, Haffajee AD, Van Der Mei HC, Haake SK, et al. Guiding periodontal pocket recolonization: A proof of concept. J Dent Res 2007;86:1078-82.

[9]. Caglar E, Kargul B, Tanboga I. Bacteriotherapy and probiotics' role on oral health. Oral Diseases 2005a;11: 131-7.Bonifait L, Chandad F, Grenier D. Probiotics for oral health: myth or reality? Journal Canadian Dental Association 2009;75:585-90. 